\title{
Elevated asymmetric dimethylarginine in young adult survivors of childhood acute lymphoblastic leukemia: A preliminary report
}

\author{
Joanna Sulicka ${ }^{\mathrm{a}, \mathrm{d}}$, Andrzej Surdacki ${ }^{\mathrm{b}, \mathrm{d}, *}$, Magdalena Strach ${ }^{\mathrm{a}, \mathrm{d}}$, Aleksander Kwater ${ }^{\mathrm{a}, \mathrm{d}}$, \\ Barbara Gryglewska ${ }^{\mathrm{a}, \mathrm{d}}$, Magdalena Ćwiklińska ${ }^{\mathrm{c}, \mathrm{e}}$, Walentyna Balwierz ${ }^{\mathrm{c}, \mathrm{e}}$ and Tomasz K. Grodzicki ${ }^{\mathrm{a}, \mathrm{d}}$ \\ ${ }^{a}$ Department of Internal Medicine and Gerontology, Faculty of Medicine, Jagiellonian University, Cracow, Poland \\ b2nd Department of Cardiology, Faculty of Medicine, Jagiellonian University, Cracow, Poland \\ ${ }^{\mathrm{c}}$ Department of Pediatric Oncology and Hematology, Polish-American Institute of Pediatrics, Faculty of Medicine, \\ Jagiellonian University, Cracow, Poland \\ ${ }^{\mathrm{d}}$ University Hospital, Cracow, Poland \\ eUniversity Children's Hospital, Cracow, Poland
}

\begin{abstract}
Background: Adult survivors of childhood malignancy are predisposed to late cardiovascular (CV) complications. Our aim was to estimate plasma levels of the endogenous nitric oxide formation inhibitor asymmetric dimethylarginine (ADMA), in long-term survivors of childhood acute lymphoblastic leukemia (ALL) treated with only chemotherapy.

Methods: ADMA and its isomer symmetric dimethylarginine (SDMA) were measured in 25 former ALL patients (aged 1828 years) who had survived without recurrent disease $\geqslant 5$ years from completing chemotherapy without cranial irradiation, and in 20 healthy controls (aged 20-31 years).

Results: Characteristics of the both groups were similar, except for lower high-density lipoproteins-cholesterol (HDL-C) in ALL survivors. Compared to controls, the former ALL patients exhibited significant, albeit small, rises in levels of ADMA (0.63 \pm 0.09 [SD] vs. $0.57 \pm 0.07 \mu \mathrm{mol} / \mathrm{L} ; p=0.016$ ), but not SDMA, with a consequently increased ADMA to SDMA ratio (1.08 \pm 0.22 vs. $0.91 \pm 0.16 ; p=0.004)$. The effect of former ALL on ADMA was attenuated (intergroup $p=0.10$ [ANCOVA]) upon adjustment for HDL-C (ADMA vs. HDL-C regression coefficient: $-0.065 \pm 0.030$ [SEM]; $p=0.03$ ).

Conclusions: ADMA is elevated in adult childhood ALL survivors, which can reflect late detrimental chemotherapy effects, partially related to minor lipid profile changes. Whether these subtle ADMA elevations might herald future CV morbidity, remains to be elucidated.
\end{abstract}

Keywords: Acute lymphoblastic leukemia, asymmetric dimethylarginine, late effects, long-term survivors of childhood malignancy

\section{Introduction}

Adult survivors of childhood and adolescence malignancy are at increased risk of late cardiovascular (CV) complications [1,2]. Acute lymphoblastic leukemia

* Corresponding author: Andrzej Surdacki, MD, FESC, $2^{\text {nd }}$ Department of Cardiology, Jagiellonian University, 17 Kopernika Street, 31-501 Cracow, Poland. Tel.: +48 12 4247180; Fax: +48 126368 039; E-mail: surdacki.andreas@gmx.net.
(ALL) is the most common pediatric malignancy and adult survivors of childhood-onset ALL account for about $28 \%$ of the Childhood Cancer Survival Study cohort [1]. Among survivors of childhood leukemia (ALL in about $90 \%$ ), the relative hazard of congestive heart failure, myocardial infarction and late-occurring stroke was increased 4.2-fold, 3.3-fold and 6.4-fold, respectively, with the reference to the sibling control groups $[3,4]$. Additionally, in ALL survivors standardized CV mortality ratio was elevated 4.2-fold compared with the age-, sex- and year-matched US population [1]. 
The excessive $\mathrm{CV}$ risk in former adult survivors of childhood ALL is ascribed to obesity, insulin resistance and related components of the metabolic syndrome (associated with physical inactivity and growth hormone $(\mathrm{GH})$ deficiency, mainly secondary to cranial irradiation, but also to chemotherapy by itself) $[2$, 5-7], low physical fitness and exercise capacity [2,8], anthracycline-induced cardiomyopathy [2], endothelial dysfunction $[9,10]$ and endothelial cell apoptosis $[10$, $11]$, as well as methotrexate-dependenthyperhomocysteinemia [2,12].

Recently, Dengel et al. [13] described impaired brachial artery flow-mediated dilation (FMD) - a noninvasive index of vasodilatory endothelial dysfunction [14, 15], closely reflecting an analogous disorder in the coronary circulation [16] - in young adult survivors of childhood-onset ALL irrespective of previous cranial irradiation. Endothelial dysfunction, largely dependent on reduced bioavailability of nitric oxide (NO), an endogenous anti-atherogenic molecule, is an antecedent of atherosclerotic plaques and adverse CV events [17].

Asymmetric dimethylarginine (ADMA), an endogenous inhibitor of NO formation, is associated with $\mathrm{CV}$ risk factors, endothelial dysfunction, early carotid atherosclerosis and adverse CV events in patients with overt atherosclerotic CV disease [18,19]. Furthermore, ADMA predicted adverse outcome over 11-24 years in low-risk population-based cohorts [20,21]. Although ADMA was elevated in adults with hematological malignancies, including ALL, prior to initiation of chemotherapy [22], ADMA concentrations in longterm ALL survivors have not been reported so far. Our aim was to estimate blood ADMA levels in young adult survivors of childhood ALL treated with only chemotherapy.

\section{Materials and methods}

\subsection{Subjects}

We studied 25 young adult patients (median age, 20 years [range, 18-28]) with ALL diagnosed during childhood who had survived without recurrent disease for a minimum of 5 years from completing chemotherapy. All the patients had been treated in a tertiary care center (Department of Pediatric Oncology and Hematology, Jagiellonian University, Cracow, Poland) according to ALL-BFM 90 protocol for the standard-risk group [23]. None of the subjects received cranial irradiation. ALL was diagnosed at the mean age of $8.4 \pm$
4.1 (SD) years; average interval from ALL diagnosis to study enrollment was $12.6 \pm 2.1$ years. The mean time elapsed since the completion of chemotherapy was 9.5 \pm 2.2 years (range, 5-13). We also recruited a control group of young healthy volunteers (median age, 22 years [range, 20-31]), mainly among the personnel of our hospital, their relatives and medical students.

Exclusion criteria were common for both the groups and included clinical evidence of atherosclerotic CV disease, heart failure, hypertension, diabetes, total cholesterol $\geqslant 6.0 \mathrm{mmol} / \mathrm{L}$, estimated glomerular filtration rate $(\mathrm{eGFR})<90 \mathrm{~mL} / \mathrm{min}$ per $1.73 \mathrm{~m}^{2}$ of bodysurface area, chronic coexistent diseases, acute infections within previous 2 weeks and high-sensitivity Creactive protein (hs-CRP) $>5 \mathrm{mg} / \mathrm{L}$. In accordance with the Declaration of Helsinki, the protocol had been approved by the ethics committee of our university and each subject gave written informed consent prior to the inclusion in the study.

\subsection{Study protocol}

The protocol was carried out in the morning in the outpatient clinic of our center (Department of Internal Medicine and Gerontology, Jagiellonian University, Cracow, Poland). The subjects had been previously asked to refrain from eating, smoking and alcohol or caffeine consumption overnight. Demographic and clinical characteristics were recorded; blood pressure was measured 3 times on the left arm after 5 min of rest in a sitting position, values from last two readings were averaged. Then a venous blood sample was drawn from a left antecubital vein for measurements of erythrocyte sedimentation rate (ESR), complete blood count, hs-CRP, serum glucose, glycosylated hemoglobin, homocysteine, total cholesterol, lowdensity lipoproteins-cholesterol (LDL-C), high-density lipoproteins-cholesterol (HDL-C), triglycerides and creatinine. We calculated eGFR by the novel Chronic Kidney Disease Epidemiology Collaboration (CKDEPI) formula which is more accurate than the Modification of Diet in Renal Disease study equation, especially at higher GFR [24].

Plasma for future determinations of ADMA and its stereoisomer symmetric dimethylarginine (SDMA) was separated and frozen at $-70^{\circ} \mathrm{C}$ until assayed. ADMA and SDMA were measured by validated [25] commercially available enzyme-linked immunosorbent assays (DLD Diagnostika GmbH, Hamburg, Germany). The lower detection limits were $0.05 \mu \mathrm{mol} / \mathrm{L}$ for ADMA and SDMA and intra-assay and inter- 
Table 1

Comparison of characteristics of ALL survivors and control subjects

\begin{tabular}{lccc}
\hline Characteristic & ALL survivors $(n=25)$ & Controls $(n=20)$ & $p$-value \\
\hline Male gender $(\%)$ & $9(36 \%)$ & $8(40 \%)$ & 0.79 \\
Smoking habit $(\%)$ & $5(20 \%)$ & $7(35 \%)$ & 0.27 \\
Height $(\mathrm{cm})$ & $170 \pm 9$ & $171 \pm 10$ & 0.82 \\
Body mass index $\left(\mathrm{kg} / \mathrm{m}^{2}\right)$ & $22.6 \pm 3.3$ & $21.8 \pm 2.3$ & 0.35 \\
Waist circumference $(\mathrm{cm})$ & $75 \pm 9$ & $73 \pm 10$ & 0.53 \\
Waist-to-hip ratio & $0.79 \pm 0.07$ & $0.76 \pm 0.08$ & 0.33 \\
Mean arterial pressure $\left(\mathrm{mmHg}^{2}\right)$ & $87 \pm 6$ & $88 \pm 6$ & 0.57 \\
eGFR, $\left(\mathrm{mL} / \mathrm{min}\right.$ per $\left.1.73 \mathrm{~m}^{2}\right)$ & $125 \pm 11$ & $120 \pm 12$ & 0.12 \\
ESR $(\mathrm{mm} / \mathrm{h})$ & $4(2-9)$ & $3(2-6)$ & 0.27 \\
Hs-CRP $(\mathrm{mg} / \mathrm{L})$ & $0.6(0.2-1.1)$ & $0.5(0.3-1.1)$ & 0.87 \\
Hemoglobin $(\mathrm{g} / \mathrm{dL})$ & $14.7 \pm 1.7$ & $14.5 \pm 1.6$ & 0.76 \\
Red blood cells $\left(10^{6} / \mu \mathrm{L}\right)$ & $5.0 \pm 0.6$ & $5.0 \pm 0.5$ & 0.66 \\
White blood cells $\left(10^{3} / \mu \mathrm{L}\right)$ & $5.9 \pm 1.4$ & $5.5 \pm 1.2$ & 0.27 \\
Platelets $\left(10^{3} / \mu \mathrm{L}\right)$ & $225 \pm 35$ & $238 \pm 42$ & 0.25 \\
LDL-C $(\mathrm{mmol} / \mathrm{L})$ & $2.3 \pm 0.6$ & $2.3 \pm 0.6$ & 0.78 \\
HDL-C $(\mathrm{mmol} / \mathrm{L})$ & $1.5 \pm 0.4$ & $1.8 \pm 0.5$ & $\mathbf{0 . 0 2}$ \\
Triglycerides $(\mathrm{mmol} / \mathrm{L})$ & $0.5(0.7-1.4)$ & $4.6 \pm 0.5$ & $0.1 .1)$ \\
Glucose $(\mathrm{mmol} / \mathrm{L})$ & $4.5 \pm 0.5$ & $5.2 \pm 0.5$ & 0.89 \\
Glycosylated hemoglobin $(\%)$ & $5.3 \pm 0.4$ & $10.3 \pm 4.4$ & 0.36 \\
Homocysteine $(\mu \mathrm{mol} / \mathrm{L})$ & $11.8 \pm 6.0$ &
\end{tabular}

Data are shown as means $\pm \mathrm{SD}$, medians (interquartile range) or $n(\%) ; p$-values $<0.05$ in bold. ALL: acute lymphoblastic leukemia; eGFR: estimated glomerular filtration rate; ESR: erythrocyte sedimentation rate; Hs-CRP: high-sensitive C-reactive protein; LDL-C: low-density lipoproteins-cholesterol; HDL-C: high-density lipoproteins-cholesterol.

assay coefficients of variation were $5.7 \%$ and $10.3 \%$ (ADMA), and $6.1 \%$ and $9.8 \%$ (SDMA). According to the manufacturer, cross-reactivity with L-arginine and other methylarginines averaged below $0.01-0.02 \%$ (SDMA or ADMA vs. L-arginine), 0.44-1.2\% (ADMA vs. SDMA), and 0.7-1.0\% (SDMA or ADMA vs. $N^{G_{-}}$ monomethyl-L-arginine).

\subsection{Statistical analysis}

Data are presented as means \pm SD unless indicated otherwise for continuous variables with normal distribution, medians and interquartile range $\left(25^{\text {th }}\right.$ and $75^{\text {th }}$ percentiles) for not normally distributed continuous parameters and numbers (proportions) for categorical data. The accordance with a normal distribution was tested by the Kolmogorov-Smirnov test. Intergroup differences were assessed by the unpaired 2-sided Student's $t$-test or Mann-Whitney $U$ test for continuous variables and Fisher's exact test for proportions.

Analysis of variance (ANOVA) and covariance (ANCOVA) were applied to estimate interaction effects between ALL history and gender or self-reported smoking on ADMA and SDMA as well as to adjust for covariates for which the $p$-value in a univariate analysis was $\leqslant 0.15$. Effects of continuous covariates were represented by non-standardized regression coefficients $(\beta$, mean $\pm \mathrm{SEM})$ corresponding to the change in a dependent variable for an increase of one unit of measurement in the covariate. Uniformity of variance was confirmed by Levene's test. Bivariate relations were assessed by Pearson's $(r$ ) or Spearman's (rho) correlation coefficients. A $p$-value $<0.05$ was inferred significant. All statistical tests were performed using STATISTICA (data analysis software system), version 9.1. (StatSoft, Inc., Tulsa, Oklahoma, USA).

\section{Results}

\subsection{Clinical and demographic subjects' characteristics}

Characteristics of the ALL survivors and the controls were similar, except for significantly lower HDL-C and insignificant tendencies to higher eGFR and lower percentage of smokers in the former ALL patients (Table 1). Two out of 25 ALL survivors exhibited the presence of 2 diagnostic traits for the metabolic syndrome by the National Cholesterol Education Program Adult Treatment Panel III revised criteria [26].

\subsection{Plasma dimethylarginines in ALL survivors}

As compared with the controls, the ALL survivors exhibited a significant, albeit small, increase in plasma 
Table 2

Plasma levels of dimethylarginines in ALL survivors and control subjects

\begin{tabular}{lccc}
\hline Variable & ALL survivors $(n=25)$ & Controls $(n=20)$ & $p$-value \\
\hline ADMA $(\mu \mathrm{mol} / \mathrm{L})$ & $0.63 \pm 0.09$ & $0.57 \pm 0.07$ & $\mathbf{0 . 0 1 6}$ \\
SDMA $(\mu \mathrm{mol} / \mathrm{L})$ & $0.60 \pm 0.10$ & $0.64 \pm 0.10$ & 0.17 \\
ADMA/SDMA ratio & $1.08 \pm 0.22$ & $0.91 \pm 0.16$ & $\mathbf{0 . 0 0 4}$ \\
\hline
\end{tabular}

Data are shown as means $\pm \mathrm{SD} ; p$-values $<0.05$ in bold. ADMA: asymmetric dimethylarginine; ALL: acute lymphoblastic leukemia; SDMA: symmetric dimethylarginine.

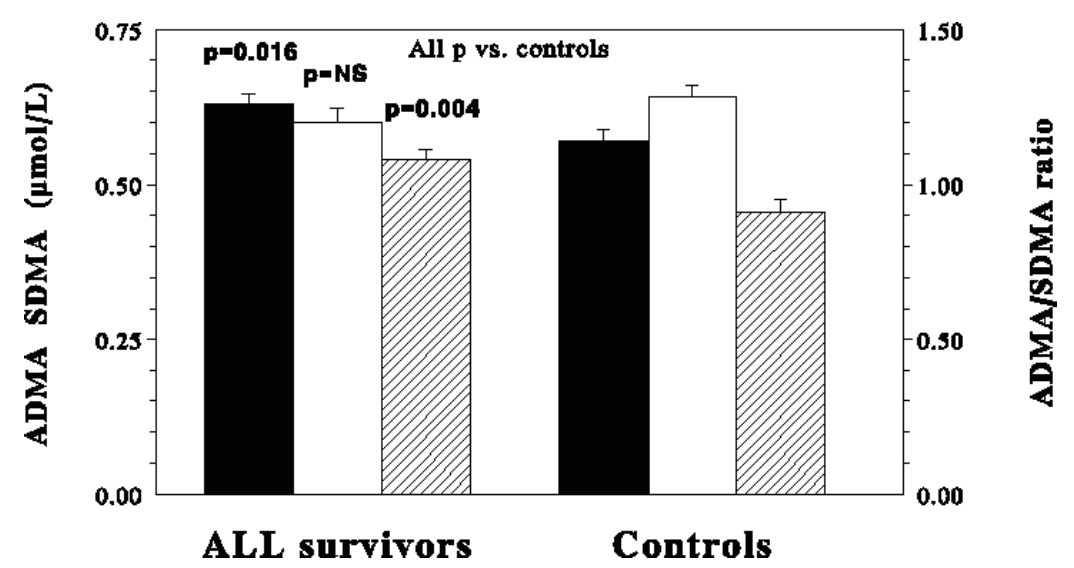

Fig. 1. Elevated plasma levels of asymmetric dimethylarginine (ADMA) (solid bars) but not symmetric dimethylarginine (SDMA) (empty bars), with a consequently increased ADMA to SDMA ratio (hatched bars) in young adult survivors of childhood acute lymphoblastic leukemia (ALL) compared with healthy controls. Values are presented as means and SEM. NS: not significant.

levels of ADMA, but not SDMA, with a consequent elevation of the ADMA to SDMA ratio (Table 2; Fig. 1). We found no interaction effects between former ALL and gender or smoking on ADMA ( $p$ for interaction: 0.75 and 0.38 , respectively, by a two-way ANOVA) and SDMA (respective $p$-values: 0.17 and 0.33 ). The results did not substantially change following exclusion of the smokers or 2 ALL survivors with 2 diagnostic traits for the metabolic syndrome.

\subsection{Correlates of plasma levels of dimethylarginines}

For the two groups pooled together, ADMA correlated moderately to reduced HDL-C $(r=-0.42, p=$ $0.005)$ and weakly to body mass index (BMI) $(r=$ $0.33 ; p=0.03)$, whereas SDMA was associated with higher creatinine $(r=0.44, p=0.003)$ and lower eGFR $(r=-0.43 ; p=0.004)$. ADMA and SDMA were mutually unrelated $(r=0.21 ; p=0.18)$ and did not correlate with the remaining demographic, clinical and biochemical features (listed in Table 1), age at ALL diagnosis, interval from ALL diagnosis and time elapsed since the completion of chemotherapy $(p>0.15)$.

The significance of the intergroup differences in ADMA was retained following inclusion of BMI as a covariate to the one-way ANCOVA. However, the ef- fect of former ALL on ADMA was attenuated (adjusted intergroup $p=0.10$ by ANCOVA) upon adjustment for HDL-C (ADMA vs. HDL-C: $\beta=-0.065 \pm 0.030$; $p=0.03$ ). The lack of between-group differences in SDMA was maintained after controlling for eGFR.

\section{Discussion}

Our salient observation was a small yet significant rise in ADMA levels in young adult survivors of childhood-onset ALL.

\subsection{Possible mechanisms of an increased plasma ADMA to SDMA ratio in ALL survivors}

Selective ADMA elevations along with unchanged SDMA levels had been previously reported in youngto-middle-aged subjects free of preexisting atherosclerotic $\mathrm{CV}$ disease but with classical risk factors: hypercholesterolemia (2-fold) [27], uncomplicated newlydiagnosed essential hypertension (2-fold) [28], highnormal blood pressure (by about 40\%) [29] and uncomplicated type 2 diabetes mellitus (2-fold) [30]. An increased plasma ADMA/SDMA ratio was linked to overexpression of type I protein arginine $N$-methyl- 
transferases (PRMTs-I) (catalyzing ADMA but not SDMA formation [18]) and/or depressed activity of dimethylarginine dimethylaminohydrolase (DDAH) (hydrolyzing over $80 \%$ of ADMA but inactive to SDMA [18]) in endothelial cells exposed to angiotensin II [31], native or oxidized LDL (oxLDL) [32,33], tumor necrosis factor- $\alpha$ [32], homocysteine [34] and high glucose concentrations [35]. Although none of the study subjects exhibited hypertension, diabetes or severe hypercholesterolemia, minor intergroup differences in patients' characteristics previously accompanied by preferential ADMA accumulation could contribute to relatively small ADMA rises in the ALL survivors. Nevertheless, the latter appears unlikely because blood pressure [28,29], LDL-C [27], homocysteine [34], glycosylated hemoglobin/glucose [30,35], triglycerides [36] and inflammatory status [37] were similar across both the groups.

However, our former ALL patients exhibited significantly lower HDL-C levels, ADMA was correlated to reduced HDL-C (in agreement with data from the largest cohort of young healthy adults with measured ADMA [38]) and this association partially explained ADMA elevations in the ALL survivors. Depressed HDL-C might facilitate ADMA accumulation because prior exposure of cultured endothelial cells to HDL counteracted the oxLDL-dependent inhibition of DDAH expression and activity, with a consequently lower ADMA level in the culture supernatant [39].

Furthermore, a lower HDL-C concentration, a part of atherogenic dyslipidemia, might indicate insulin resistance, known to be associated with elevated ADMA [40]. Admittedly, metabolic sensitivity to insulin was not quantified in the present study. On the other hand, none of our patients met the metabolic syndrome criteria [26], exclusion of 2 former ALL patients with 2 metabolic syndrome diagnostic traits produced similar results, and waist circumference, waist-to-hip ratio, BMI and triglycerides did not differ between the groups. These findings were presumably due to the a priori exclusion of the patients treated with cranial irradiation, a major factor responsible for subsequent $\mathrm{GH}$ deficiency and late insulin resistance [6]. Nevertheless, insulin resistance, exhibited by long-term ALL survivors after combined chemotherapy and cranial radiotherapy, is also present - even though less pronouncedin those treated with chemotherapy alone [5].

In addition, as cultured endothelial cells release more free ADMA than SDMA [41] - both of which are liberated during breakdown of proteins containing dimethylated arginine residues [18] - enhanced propensity to apoptosis and accelerated endothelial cell turnover could also increase the ADMA/SDMA ratio. Anthracyclines induced endothelial apoptosis [10,11] (a correlate of endothelial dysfunction accompanying aging [42]) and subsequently impaired endotheliumdependent relaxation in rabbit mesenteric arteries [10], which was implied as a probable basis for decreased FMD in 14 young cancer survivors who had completed chemotherapy 2 to 60 months before [9].

Over 2-fold selective ADMA elevations were recently reported in adults with hematological malignancies, including ALL, before onset of chemotherapy [22]. This finding was hypothetically linked with increased turnover of tumor cells, extensive protein catabolism and inflammatory activation [22], neither of which was operative in our asymptomatic long-term survivors of childhood ALL. Nevertheless, we are not able to exclude persistent alterations of ADMA metabolism initiated by a direct interference of chemotherapeutic agents administered during childhood with cellular pathways affecting ADMA elaboration.

\subsection{Preferential ADMA accumulation - a marker of endothelial oxidative stress?}

Keeping in mind that at a normal function of the kidney - the predominant route of SDMA elimination [18] - a higher ADMA/SDMA ratio can be perceived as a rough indicator of reduced activity of DDAH [30], a redox-sensitive enzyme decomposing ADMA [43], we can set forth the speculative concept of excessive intracellular oxidative stress as an early endothelial abnormality in our young ALL survivors without renal pathology. Then a minor increase of ADMA may be a starting point for its further gradual elevations [43]. Accordingly, ADMA may contribute to uncoupling of endothelial NO synthase (eNOS), i.e., dissociation of oxygen reduction by the reductase domain from L-arginine oxidation by the oxygenase domain, which converts eNOS from a source of NO into a superoxide generator [41,43-46]. Superoxide reacts avidly with NO to form peroxynitrite that oxidizes tetrahydrobiopterin, an essential eNOS cofactor, thus perpetuating eNOS uncoupling $[43,46]$. The presented sequence of events increases intracellular oxidative stress that in turn further promotes ADMA formation via increased PRMTs-I expression [33] and/or reduced DDAH activity [43]. This feed-forward reaction is presumably interwoven with other positive feedback loops with likely involvement of the local renin-angiotensin system, endothelial membrane-bound nicotinamide ade- 
nine dinucleotide phosphate (NADPH) oxidases and the oxidant-sensitive nuclear factor- $\kappa \mathrm{B}$-regulated proinflammatory transcription pathway [31,41,46,47], all of which converge to promote endothelial dysfunction and monocyte-endothelial interactions, early steps in atherogenesis. A recent experimental study by Jacobi et al. [48] provided proof-of-principle that ADMA more than a disease marker - is one of culprit molecules in atherogenesis.

\subsection{Can small ADMA elevations be a very early herald of late $C V$ morbidity in long-term ALL survivors?}

Regardless of the mechanisms involved in preferential accumulation of ADMA over SDMA in young adult survivors of childhood ALL, a key question is whether this small rise in ADMA may translate to future adverse $\mathrm{CV}$ events. However, in 1,808 healthy participants of the Cardiovascular Risk in Young Finns Study without atherosclerotic $\mathrm{CV}$ disease, baseline ADMA was predictive of peripheral endothelial dysfunction measured after 6 years and the multivariate longitudinal inverse association between ADMA and endotheliumdependent vasodilation appeared stronger than in crosssectional analysis [49]. Moreover, in 880 middle-aged women recruited to the Population Study of Women in Gothenburg in the late 1960s, those in the top quintile of baseline ADMA exhibited a cumulative relative hazard of myocardial infarction and stroke of 1.75 compared with the bottom four-fifths over a 24-year followup [21]. Importantly, separation of the cumulative risk curves for these $\mathrm{CV}$ events was observed not earlier than at about 13 years [21]. It is noteworthy that each $0.15 \mu \mathrm{mol} / \mathrm{L}$ increase in ADMA (by $1 \mathrm{SD}$ ) was associated with a $30 \%$ increase in incident myocardial infarction and stroke [21], which suggests potential clinical importance of relatively small changes in ADMA. Therefore it may be hypothesized that in young healthy subjects even minor ADMA elevations within the normal range, as in our ALL survivors, might be a hallmark of early abnormalities which - on a long-term basis - can lead to clinically overt atherosclerosis.

\subsection{Limitations of the study}

Several limitations to this study need to be acknowledged. First, a small number of the participants inevitably constrains the conclusions based on only a single measurement of dimethylated L-arginine analogues. Moreover, we are fully aware of the fact that repeated assays of ADMA and SDMA over time would strengthen prognostic implications of our findings with regard to the risk of future $\mathrm{CV}$ events. However, contrary to previous reports of endothelial dysfunction in childhood malignancy survivors $[9,13,50]$, we found elevated ADMA, a biochemical marker of impaired endothelial function, in the former ALL patients who had been treated with a uniform chemotherapy protocol, which was likely to limit at least one source of data variability. Second, the percentage of selfreported smokers was insignificantly higher in the control group. Smoking is associated with endothelial dysfunction [14] and there are inconsistent data on the relationship between smoking and ADMA levels [1921,38,51-53]. Nevertheless, we demonstrated neither a significant effect of smoking on ADMA, nor interactions between smoking status and ALL history, which could result from a probably relatively short exposure to tobacco smoke in the young study participants. Third, we did not exclude GH deficiency, a condition associated with selective rises in ADMA and unchanged SDMA levels [54]. Although GH deficiency was linked predominantly to cranial radiotherapy [6], yet hypothalamic-pituitary insufficiency may occur also in long-term survivors of childhood cancer treated with no cranial or total body irradiation [6,7]. On the other hand, subjects' height was similar in both our groups, which argues against considerable impairment of the GH-dependent axis in the ALL survivors.

\section{Conclusions}

ADMA is slightly elevated in young adult survivors of childhood ALL. This preliminary finding can reflect late detrimental effects of chemotherapy on the vascular endothelium or other as yet unknown target cells, in part related to minor changes in the lipid profile. Whether these subtle ADMA elevations might be a very early herald of future CV morbidity, remains to be elucidated in a prospective study.

\section{Acknowledgements}

This study was partially supported by a research grant (No. K/ZDS/001475) from the Polish State Committee for Scientific Research, Warsaw, Poland. A part of data of a subgroup of our subjects was presented as a poster at the European Society of Cardiology Congress, Paris, France, August 2011 and its abstract has been published in the Abstract Supplement to the European Heart Journal. 


\section{References}

[1] A.C. Mertens, Q. Liu, J.P. Neglia et al., Cause-specific late mortality among 5-year survivors of childhood cancer: the Childhood Cancer Survivor Study, J Natl Cancer Inst 100 (2008), 1368-1379.

[2] K.C. Oeffinger, Are survivors of acute lymphoblastic leukemia (ALL) at increased risk of cardiovascular disease?, Pediatr Blood Cancer 50(2 Suppl) (2008), 462-467.

[3] D.A. Mulrooney, M.W. Yeazel, T. Kawashima et al., Cardiac outcomes in a cohort of adult survivors of childhood and adolescent cancer: retrospective analysis of the Childhood Cancer Survivor Study cohort, BMJ 339 (2009), b4606.

[4] D.C. Bowers, Y. Liu, W. Leisenring et al., Late-occurring stroke among long-term survivors of childhood leukemia and brain tumors: a report from the Childhood Cancer Survivor Study, J Clin Oncol 24 (2006), 5277-5282.

[5] K.C. Oeffinger, B. Adams-Huet, R.G. Victor et al., Insulin resistance and risk factors for cardiovascular disease in young adult survivors of childhood acute lymphoblastic leukemia, $J$ Clin Oncol 27 (2009), 3698-3704.

[6] J.G. Gurney, K.K. Ness, S.D. Sibley et al., Metabolic syndrome and growth hormone deficiency in adult survivors of childhood acute lymphoblastic leukemia, Cancer 107 (2006), 1303-1312.

[7] S.R. Rose, R.E. Schreiber, N.S. Kearney et al., Hypothalamic dysfunction after chemotherapy, J Pediatr Endocrinol Metab 17 (2004), 55-66.

[8] L.S. Järvelä, H. Niinikoski, P.M. Lähteenmäki et al., Physical activity and fitness in adolescent and young adult longterm survivors of childhood acute lymphoblastic leukaemia, $J$ Cancer Surviv 4 (2010), 339-345.

[9] A.Y. Chow, C. Chin, G. Dahl and D.N. Rosenthal, Anthracyclines cause endothelial injury in pediatric cancer patients: A pilot study, J Clin Oncol 24 (2006), 925-928.

[10] T. Murata, H. Yamawaki, R. Yoshimoto et al., Chronic effect of doxorubicin on vascular endothelium assessed by organ culture study, Life Sci 69 (2001), 2685-2695.

[11] S. Wu, Y. Ko, M. Teng et al., Adriamycin-induced cardiomyocyte and endothelial cell apoptosis: in vitro and in vivo studies, J Mol Cell Cardiol 34 (2002), 1595-1607.

[12] H. Refsum, F. Wesenberg and P.M. Ueland, Plasma homocysteine in children with acute lymphoblastic leukemia: changes during a chemotherapeutic regimen including methotrexate, Cancer Res 51 (1991), 828-835.

[13] D.R. Dengel, K.K. Ness, S.P. Glasser, E.B. Williamson, K.S. Baker and J.G. Gurney, Endothelial function in young adult survivors of childhood acute lymphoblastic leukemia, $J$ Pediatr Hematol Oncol 30 (2008), 20-25.

[14] D.S. Celermajer, K.E. Sorensen, V.M. Gooch et al., Noninvasive detection of endothelial dysfunction in children and adults at risk of atherosclerosis, Lancet 340 (1992), 11111115 .

[15] E.H. Lieberman, M.D. Gerhard, A. Uehata et al., Flowinduced vasodilation of the human brachial artery is impaired in patients $<40$ years of age with coronary artery disease, $A m$ J Cardiol 78 (1996), 1210-1214.

[16] T.J. Anderson, A. Uehata, M.D. Gerhard et al., Close relation of endothelial function in the human coronary and peripheral circulations, J Am Coll Cardiol 26 (1995), 1235-1241.

[17] V. Schächinger, M.B. Britten and A.M. Zeiher, Prognostic impact of coronary vasodilator dysfunction on adverse long-term outcome of coronary heart disease, Circulation 101 (2000), 1899-1906.
[18] P. Vallance and J. Leiper, Cardiovascular biology of the asymmetric dimethylarginine: dimethylarginine dimethylaminohydrolase pathway, Arterioscler Thromb Vasc Biol 24 (2004), 1023-1030.

[19] R.H. Böger, R. Maas, F. Schulze and E. Schwedhelm, Asymmetric dimethylarginine (ADMA) as a prospective marker of cardiovascular disease and mortality - An update on patient populations with a wide range of cardiovascular risk, Pharmacol Res 60 (2009), 481-487.

[20] R.H. Böger, L.M. Sullivan, E. Schwedhelm et al., Plasma asymmetric dimethylarginine and incidence of cardiovascular disease and death in the community, Circulation 119 (2009), 1592-1600.

[21] T. Leong, D. Zylberstein, I. Graham et al., Swedish-IrishNorwegian Collaboration, Asymmetric dimethylarginine independently predicts fatal and nonfatal myocardial infarction and stroke in women: 24-year follow-up of the population study of women in Gothenburg, Arterioscler Thromb Vasc Biol 28 (2008), 961-967.

[22] A. Szuba, A. Chachaj, T. Wróbel et al., Asymmetric dimethylarginine in hematological malignancies: A preliminary study, Leuk Lymphoma 49 (2008), 2316-2320.

[23] M. Schrappe, A. Reiter, W.D. Ludwig et al., Improved outcome in childhood acute lymphoblastic leukemia despite reduced use of anthracyclines and cranial radiotherapy: Results of trial ALL-BFM 90. German-Austrian-Swiss ALL-BFM Study Group, Blood 95 (2000), 3310-3322.

[24] A.S. Levey, L.A. Stevens, C.H. Schmid et al., CKD-EPI (Chronic Kidney Disease Epidemiology Collaboration). A new equation to estimate glomerular filtration rate, Ann Intern Med 150 (2009), 604-612.

[25] F. Schulze, R. Wesemann, E. Schwedhelm et al., Determination of asymmetric dimethylarginine (ADMA) using a novel ELISA assay, Clin Chem Lab Med 42 (2004), 1377-1383.

[26] S.M. Grundy, J.I. Cleeman, S.R. Daniels et al., Diagnosis and management of the metabolic syndrome: An American Heart Association/National Heart, Lung, and Blood Institute Scientific Statement, Circulation 112 (2005), 2735-2752.

[27] R.H. Böger, S.M. Bode-Böger, A. Szuba et al., Asymmetric dimethylarginine (ADMA): a novel risk factor for endothelial dysfunction: its role in hypercholesterolemia, Circulation 98 (1998), 1842-1847.

[28] A. Surdacki, M. Nowicki, J. Sandmann et al., Reduced urinary excretion of nitric oxide metabolites and increased plasma levels of asymmetric dimethylarginine in men with essential hypertension, J Cardiovasc Pharmacol 33 (1999), 652-658.

[29] H. Päivä, J. Laakso, H. Laine, R. Laaksonen, J. Knuuti and O.T. Raitakari, Plasma asymmetric dimethylarginine and hyperemic myocardial blood flow in young subjects with borderline hypertension or familial hypercholesterolemia, J Am Coll Cardiol 40 (2002), 124-127.

[30] F. Abbasi, T. Asagami, J.P. Cooke et al., Plasma concentrations of asymmetric dimethylarginine are increased in patients with type 2 diabetes mellitus, Am J Cardiol 88 (2001), 1201-1203.

[31] M.F. Chen, X.M. Xie, T.L. Yang et al., Role of asymmetric dimethylarginine in inflammatory reactions by angiotensin II, J Vasc Res 44 (2007), 391-402.

[32] A. Ito, P.S. Tsao, S. Adimoolam, M. Kimoto, T. Ogawa and J.P. Cooke, Novel mechanism for endothelial dysfunction: dysregulation of dimethylarginine dimethylaminohydrolase, Circulation 99 (1999), 3092-3095.

[33] R.H. Böger, K. Sydow, J. Borlak et al., LDL cholesterol upregulates synthesis of asymmetrical dimethylarginine in hu- 
man endothelial cells: involvement of S-adenosylmethioninedependent methyltransferases, Circ Res 87 (2000), 99-105.

[34] M.C. Stühlinger, P.S. Tsao, J.H. Her, M. Kimoto, R.F. Balinta and J.P. Cooke, Homocysteine impairs the nitric oxide synthase pathway: Role of asymmetric dimethylarginine, Circulation 104 (2001), 2569-2575.

[35] K.Y. Lin, A. Ito, T. Asagami et al., Impaired nitric oxide synthase pathway in diabetes mellitus: Role of asymmetric dimethylarginine and dimethylarginine dimethylaminohydrolase, Circulation 106 (2002), 987-992.

[36] P. Lundman, M.J. Eriksson, M. Stühlinger, J.P. Cooke, A. Hamsten and P. Tornvall, Mild-to-moderate hypertriglyceridemia in young men is associated with endothelial dysfunction and increased plasma concentrations of asymmetric dimethylarginine, J Am Coll Cardiol 38 (2001), 111-116.

[37] C. Antoniades, M. Demosthenous, D. Tousoulis et al., Role of asymmetrical dimethylarginine in inflammation-induced endothelial dysfunction in human atherosclerosis, Hypertension 58 (2011), 93-98.

[38] M. Juonala, J.S. Viikari, G. Alfthan et al., Brachial artery flowmediated dilation and asymmetrical dimethylarginine in the Cardiovascular Risk in Young Finns Study, Circulation 116 (2007), 1367-1373.

[39] Z.Y. Peng, S.P. Zhao, B.M. He, D.Q. Peng and M. Hu, Protective effect of HDL on endothelial NO production: the role of DDAH/ADMA pathway, Mol Cell Biochem 351 (2011), 243-249.

[40] M.C. Stühlinger, F. Abbasi, J.W. Chu, C. Lamendola, T.L. McLaughlin and J.P. Cooke, Relationship between insulin resistance and an endogenous nitric oxide synthase inhibitor, JAMA 287 (2002), 1420-1426.

[41] R.H. Böger, S.M. Bode-Böger, P.S. Tsao, P.S. Lin, J.R. Chan and J.P. Cooke, An endogenous inhibitor of nitric oxide synthase regulates endothelial adhesiveness for monocytes, $J \mathrm{Am}$ Coll Cardiol 36 (2000), 2287-2295.

[42] K. Asai, R.K. Kudej, Y.T. Shen et al., Peripheral vascular endothelial dysfunction and apoptosis in old monkeys, Aterioscler Throm Vasc Biol 20 (2000), 1493-1499.

[43] F. Palm, M.L. Onozato, Z. Luo and C.S. Wilcox, Dimethylarginine dimethylaminohydrolase (DDAH): expression, regulation, and function in the cardiovascular and renal systems, Am J Physiol Heart Circ Physiol 293 (2007), H3227-H3245.

[44] C. Antoniades, C. Shirodaria, P. Leeson et al., Association of plasma asymmetrical dimethylarginine (ADMA) with elevated vascular superoxide production and endothelial nitric oxide synthase uncoupling: Implications for endothelial function in human atherosclerosis, Eur Heart J 30 (2009), 1142-1150.

[45] L.J. Druhan, S.P. Forbes, A.J. Pope, C.A. Chen, J.L. Zweier and A.J. Cardounel, Regulation of eNOS-derived superoxide by endogenous methylarginines, Biochemistry 47 (2008), 7256-7263.

[46] T. Teerlink, Z. Luo, F. Palm and C.S. Wilcox, Cellular ADMA: regulation and action, Pharmacol Res 60 (2009), 448-460.

[47] Z. Veresh, A. Racz, G. Lotz and A. Koller, ADMA impairs nitric oxide-mediated arteriolar function due to increased superoxide production by angiotensin II-NAD $(\mathrm{P}) \mathrm{H}$ oxidase pathway, Hypertension 52 (2008), 960-966.

[48] J. Jacobi, R. Maas, A.J. Cardounel et al., Dimethylarginine dimethylaminohydrolase overexpression ameliorates atherosclerosis in apolipoprotein E-deficient mice by lowering asymmetric dimethylarginine, Am J Pathol 176 (2010), 2559-2570.

[49] H. Päivä, M. Kähönen, T. Lehtimäki et al., Levels of asymmetrical dimethylarginine are predictive of brachial artery flowmediated dilation 6 years later. The Cardiovascular Risk in Young Finns Study, Atherosclerosis 212 (2010), 512-515.

[50] S. Zelcer, B. Chen, J. Mangel et al., Impaired vascular function in asymptomatic young adult survivors of Hodgkin lymphoma following mediastinal radiation, J Cancer Surviv 4 (2010), 218-224.

[51] R. Maas, F. Schulze, J. Baumert et al., Asymmetric dimethylarginine, smoking, and risk of coronary heart disease in apparently healthy men: prospective analysis from the populationbased Monitoring of Trends and Determinants in Cardiovascular Disease/Kooperative Gesundheitsforschung in der Region Augsburg study and experimental data, Clin Chem 53 (2007), 693-701.

[52] A. Meinitzer, U. Seelhorst, B. Wellnitz et al., Asymmetrical dimethylarginine independently predicts total and cardiovascular mortality in individuals with angiographic coronary artery disease (the Ludwigshafen Risk and Cardiovascular Health study), Clin Chem 53 (2007), 273-283.

[53] R. Schnabel, S. Blankenberg, E. Lubos et al., Asymmetric dimethylarginine and the risk of cardiovascular events and death in patients with coronary artery disease: results from the AtheroGene Study, Circ Res 97 (2005), e53-e59.

[54] K. Krzyzanowska, F. Mittermayer, C. Schnack, M. Hofer, M. Wolzt and G. Shernthaner, Circulating ADMA concentrations are elevated in hypopituitary adults with and without growth hormone deficiency, Eur J Clin Invest 35 (2005), 208-213. 


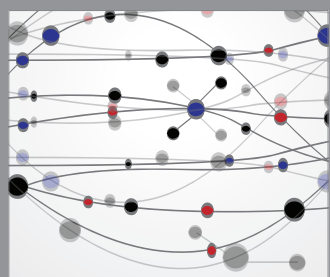

The Scientific World Journal
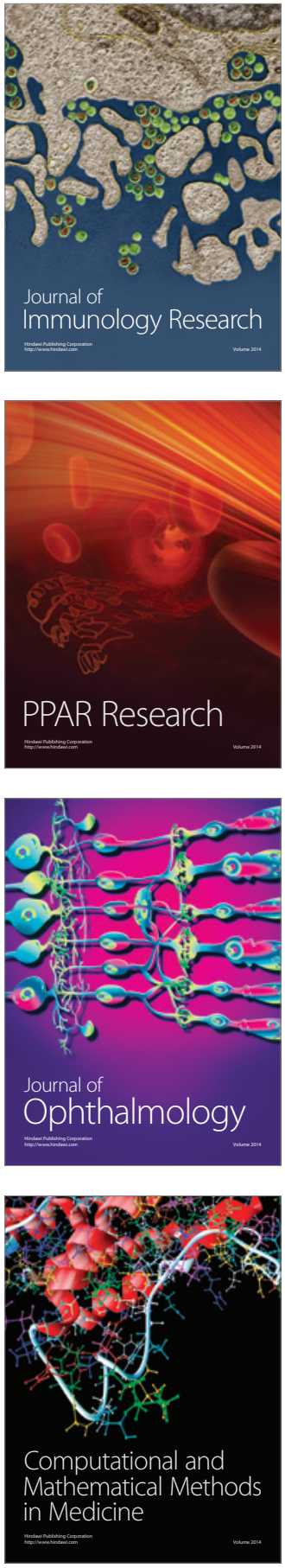

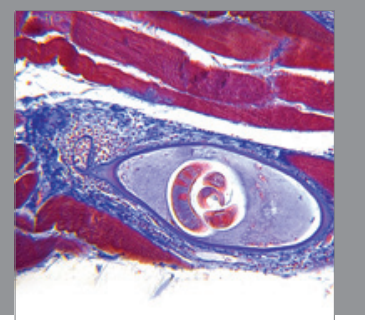

Gastroenterology

Research and Practice
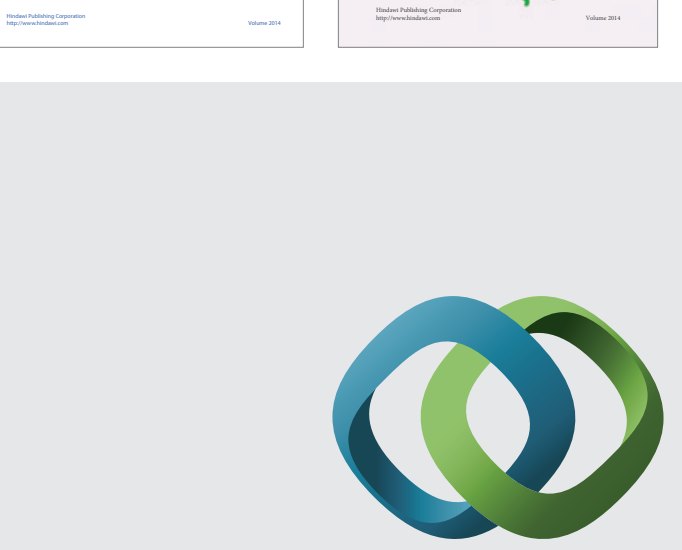

\section{Hindawi}

Submit your manuscripts at

http://www.hindawi.com
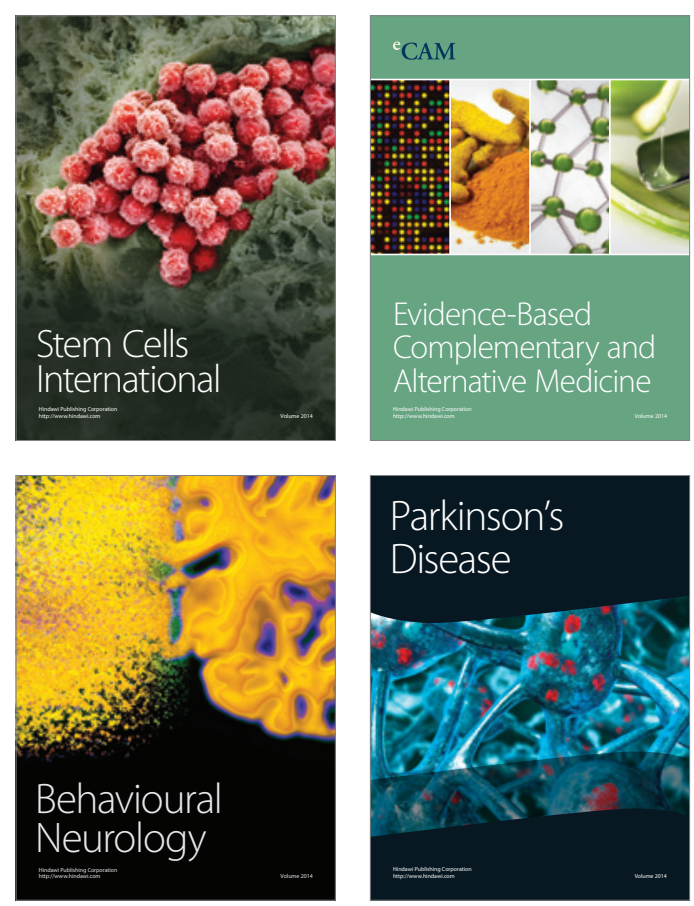

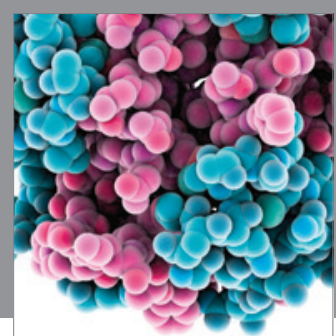

Journal of
Diabetes Research

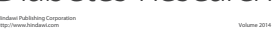

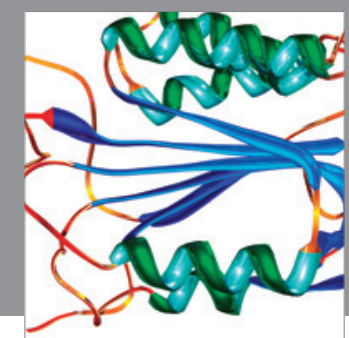

Disease Markers
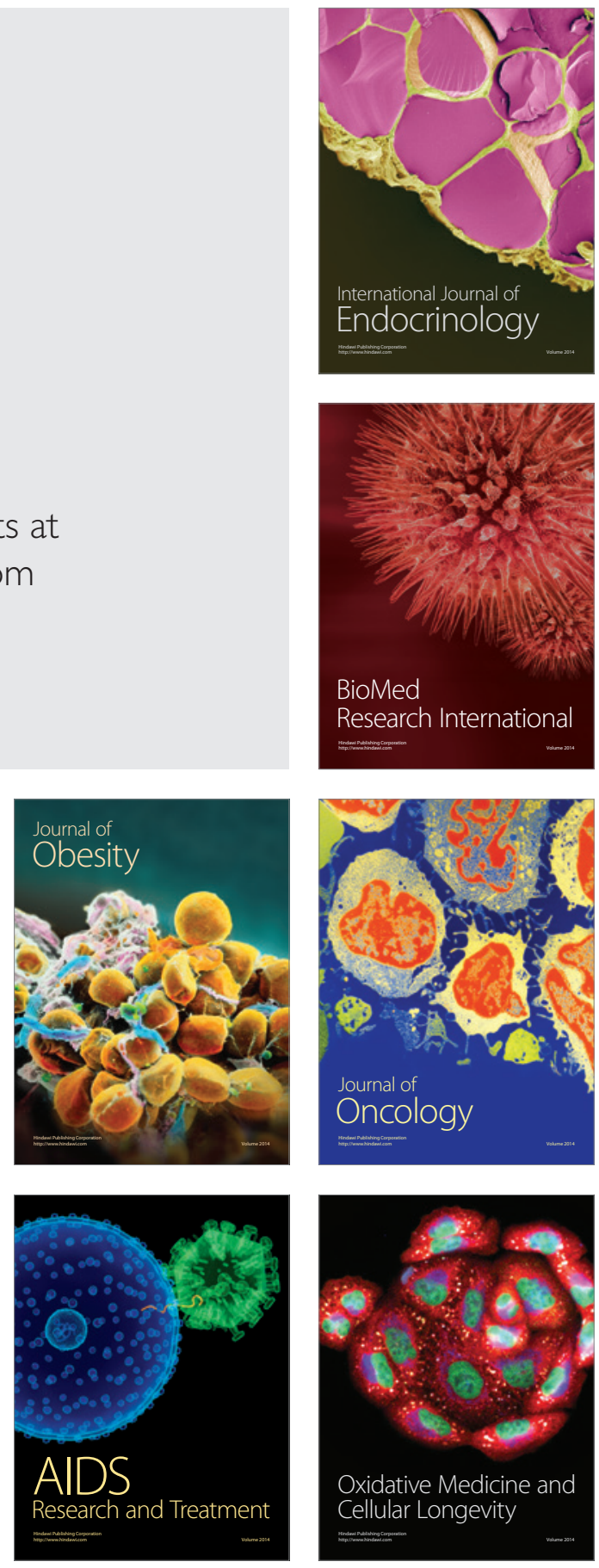